\title{
The Cultural Area of The Suicidal Behaviour
}

\author{
Gavril Cornutiu \\ Clinic of Psychiatry, University of Oradea \\ Faculty of Medicine and Pharmacy Oradea, Romania \\ Oana Cornutiu \\ Clinic of Psychiatry, University of Oradea \\ Faculty of Medicine and Pharmacy Oradea, Romania
}

\begin{abstract}
There is a cultural fluctuation of suicide. This is known since Durkheim. For this study the authors tried to outline a cultural parameter which influences suicide. Research on a group of 602,206 inhabitants in a romanian county (Bihor) of two nationalities: $\mathbf{7 0 . 9 2 \%}$ Romanians and $29.05 \%$ Hungarians has been carried out. The suicide rates between 01.01.1994 and 31.12.2003 have been analyzed. The data used came from official records - the County Forensic Pathology Laboratory. Suicide rates on distinctive ethnic areas measured in cases per 100,000 population were as follows: rural areas - 34.8 cases in 100,000 population in Hungarians and 3.96 cases in 100,000 population in Romanians; urban areas - 34.37 per 100,000 in Hungarians and 6.55 per 100,000 in Romanians; areas with mixed population (Romanians and Hungarians) $1 / 1$ - rate of suicide $1 / 1$ (16.58). In conclusion, there are Areas of Cultural Vulnerability to Suicide that require the setting up of Principles of Cultural Prophylaxis of Suicide.
\end{abstract}

Keywords: suicidal behaviour, cultural fluctuation, Area of Cultural Vulnerability, cultural prophylaxis of suicide.

\section{INTRODUCTION}

Optional human behaviour is a continuative development of automatic ethologic behaviour [1]. For instance, this truth is obvious for instinctive behaviour (i.e. feeding, reproducing etc.). All other types of behaviour are social and spiritual developments which spring from the biological necessities enrooted in us. But development may make them become so different from our organic needs, that the intermediation made by different stages of attitudinal and behavioral state may make the connection simply impossible. As they become less biological, these types of behaviour become more and more dependent on the spiritual values, determining the attitudinal states with behavioral consequences $[2,3,4]$.

\section{LITERATURE REVIEW}

Suicidal behaviour is such an example. It depends on the spiritual values of a certain place and time according to the demonstration of suicidology classic E. Durkheim [5] almost 100 years ago. From a historical point of view, there are few countries that have rigorous data about suicide [6]. Even today, not all countries reveal rigorous statistics. Despite their limitation, these statistics provide a clearly enough evolutionary tendency of the general and geographical specifics [7]. Both old and new statistics show a striking difference between countries and even regions of the same country [8]. In 1990, Romania had a suicide rate of 11.4 cases in 100,000 population. In 1996 the suicide rate in Romania was of 21.3 cases in 100,000 in men (M) and 
4.3 cases in 100,000 in women (W), with an average of about $12.8 / 100,000$. Today in Romania the difference of suicide rates between provinces varies between 6 and 18 cases/100,000.

The marked difference between areas with catholic religious frequency and those of protestant frequency is already classical. Even more, in the past it became obvious that there were big differences between locations of the same religious and cultural background. Apart from what has been discussed above, another factor is the role of the collective mentality of certain groups of population, that of the We-ity groups. Therefore, about $1 / 5$ (19\%) of suicides occur in people who do not suffer from any psychiatric disease [5].

\section{Purpose}

The purpose of this study is to analyze the profile of the suicidal behaviour of a larger population group, made up of culturally distinctive subgroups, in a geographical location, in the same time frame in order to find a cultural parameter which influences suicide.

\section{MATERIAL AND METHOD}

The studied population group was taken from Bihor County, Romania, region having 635,290 inhabitants in 1994 and 602,206 inhabitants in 2002 (the last census), the decrease being due to massive emigration after 1990 . This county is situated in North-West Romania, close to the Hungarian border. The ethnical structure is as follows: 70.92\% Romanians (R), 29.05\% Hungarians $(\mathrm{H})$ and $0.03 \%$ other nationalities. Female/Male ratio was of $51.38 \%$ / $48.62 \%$ in 2003. All suicides (886: $488 \mathrm{R} ; 402 \mathrm{H}$ ) committed in this area between 01.01.1994 and 31.12. 2003 (10 years) have been analyzed. The data were obtained from official documents of Bihor County Forensic Pathology Laboratory. Data gathered was: frequency, sex, nationality, age, locality (population group categories and distribution), method of suicide, the existence or nonexistence of a psychiatric disease, the existence of attempted suicide prior to suicide. Because of the fact that one suicide rate is a multiple of the other, there was no need for a statistical, mathematical analysis.

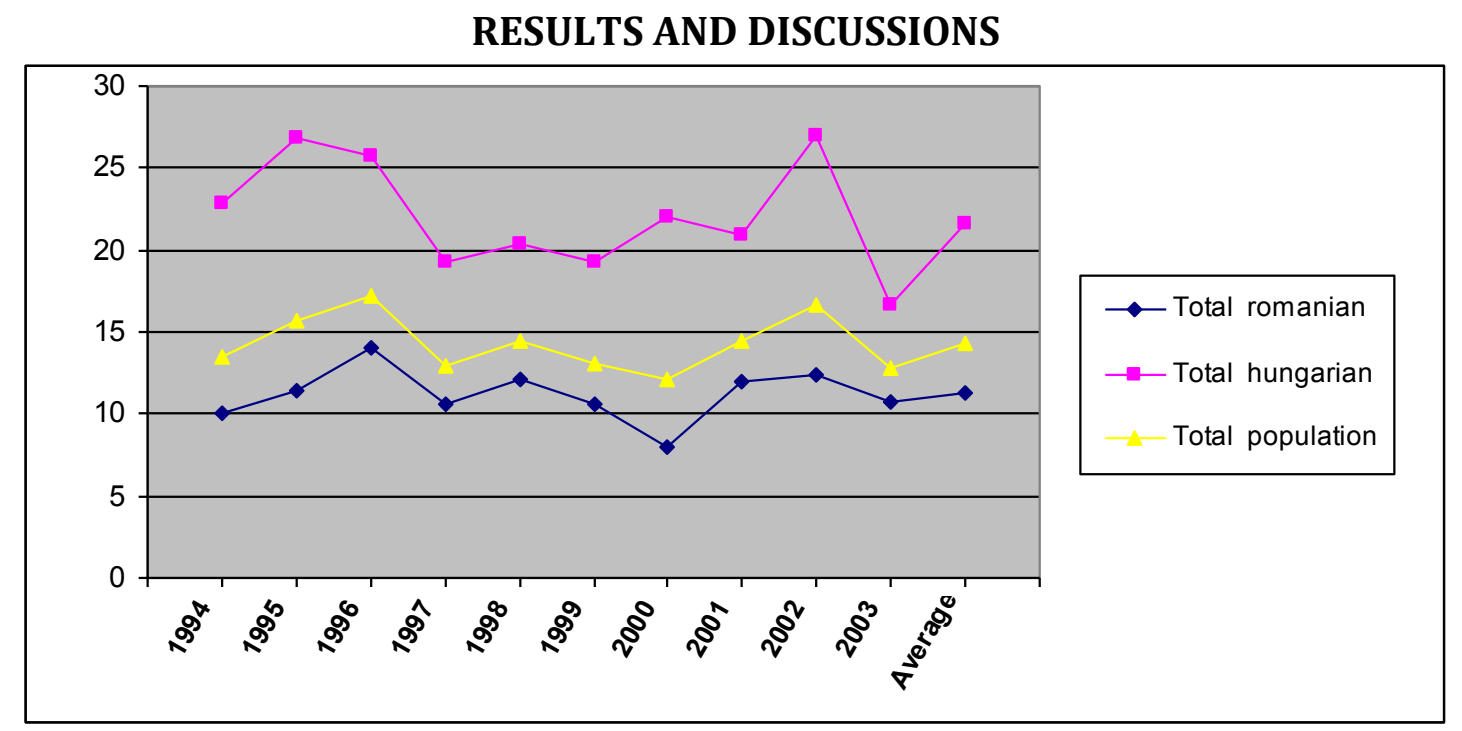

Fig. 1 The dynamics of suicide rates in groups of population by year and average

The suicide rates in Figure 1 are the rates of cases gathered from Bihor County, Romania for every year studied (from 1994 to 2003) by ethnicity measured in cases per 100,000 population.

From Figure 1, a big difference between the suicidal rate in Romanian (R) and Hungarian $(\mathrm{H})$ 
population can be observed. The average global rate, over the 10 years, was of $21.651 / 100,000$ in $\mathrm{H}$ and 11.306/100,000 in R, almost doubled in $\mathrm{H}$ than in R. Over the 10 years, the fluctuation of the suicidal rate in Hungarian population varies between 16.63 and 12.29 (1.62 times) and in the Romanian population between 8.02 and 12.29 (1.53 times). Living in the same geographical area, under the same administration, both Romanian and Hungarian people are subjected to the same social, economical and political stress levels. We call the people's suicidal reaction to this type of stress the "suicidal resonance". The suicidal resonance to situations is similar in both populations. Therefore, situational suicidal resonance cannot explain the big difference between the two suicide rates. The curve of the suicide rate in both ethnic groups has similar ups and downs. The difference consists only in the size of the ratio.

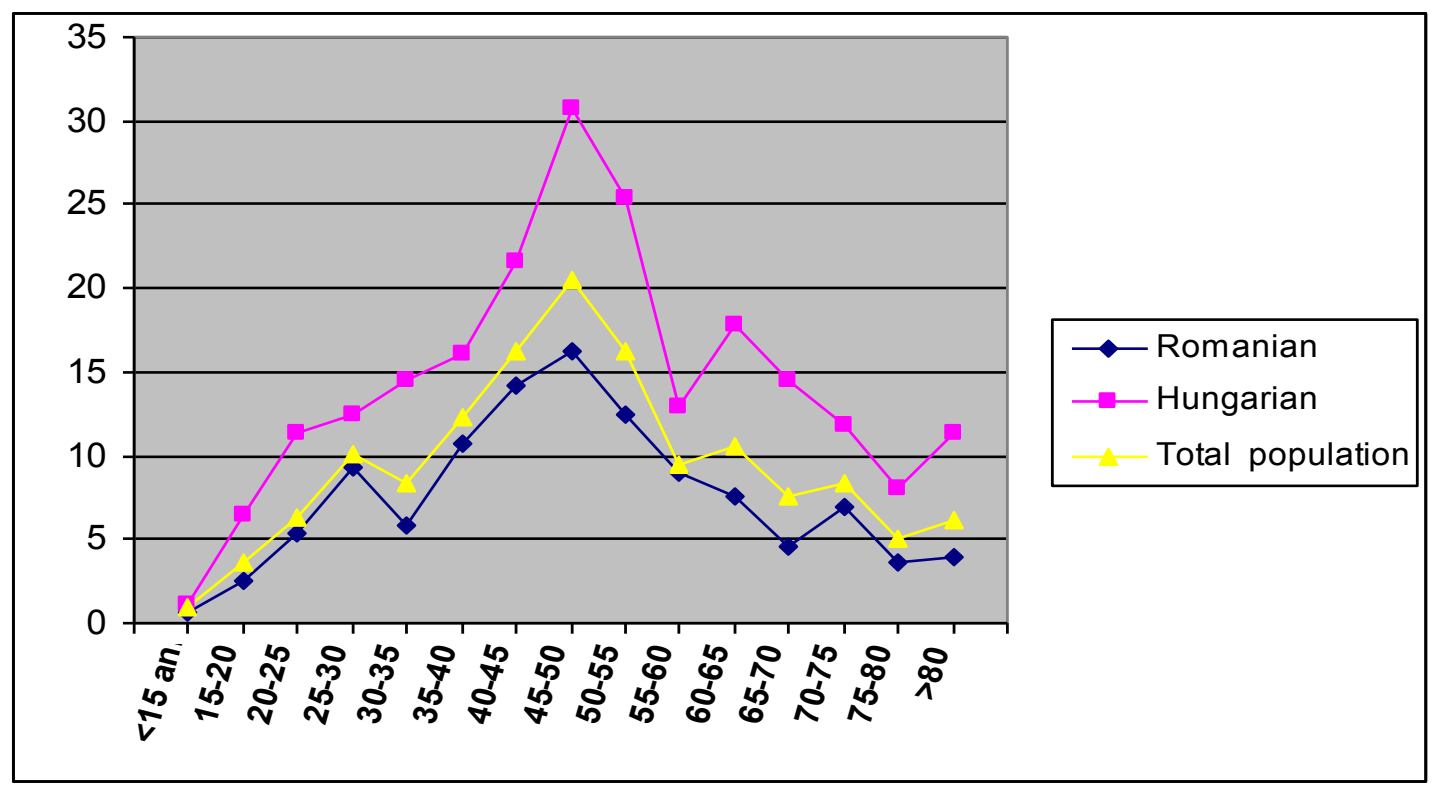

Fig. 2 The dynamics of suicide rates by age groups and groups of population

Figure 2 shows the different suicide rates by age groups for every ethnicity as well as for the total population of Bihor County, Romania for the 10 year period that was studied expressed in cases per 100,000 population.

The curves of the suicidal rate, according to age groups (Figure 2), show a similar tendency of the dynamics of the phenomenon according to age, with the same difference between suicide rates in $\mathrm{R}$ and $\mathrm{H}$ population regardless of age (a similar global tendency) $[9,10]$. Suicide peaks are noted at the age of the three existential balances: the 25-30 age group, the marriage completion period in general population, the 45-50 age group, the critical hormonal period and the biological balance, and the 60-75 age group, the retirement age. Vulnerability in the last two age groups also has a biological component.

Nevertheless, all these vulnerable factors in the general population have similar expressions in suicide rate in $\mathrm{R}$ as well as in $\mathrm{H}$. However, the difference between the final suicide rates between the two nationalities could not be explained by these parameters.

Regarding known alcoholics from the lot $(12.453 \% \mathrm{R}$ and $13.442 \% \mathrm{H})$ the psychiatric patient to suicidal ratio is almost the same. This points out that psychiatric diseases cannot be held responsible for the high suicide rates in $\mathrm{H}$ population. Alcohol intake at the time of the suicidal act has an almost identical percentage in both ethnicities: $51 \% \mathrm{R}$ and $49 \% \mathrm{H}$. In conclusion, in 1 
out of 2 people alcohol favored the suicidal act regardless of ethnicity. Rough methods such as hanging, defenestration, shooting, drowning, self arson, electrocuting and wounds are preferred by the $\mathrm{H}$ population while the softer methods (e.g. self poisoning) are more frequently used by R. We already know $[3,8,11]$ that accomplishment methods are learned by imitation. These are suicidal trends in a certain geographical area within a given population, similar with those observed by other authors $[12,13,14]$. The two groups of population in this study have different suicidal trends. In the collective mentality of the two populations there are different outlooks about methods of suicide accomplishment.

Table 1. The suicide rate on ethnic areas over the 10 studied years

\begin{tabular}{|c|c|c|c|c|c|}
\hline H Villages ${ }^{s}$ & R Villages $\%$ & $\begin{array}{r}\text { Village } \\
\text { po }\end{array}$ & $\begin{array}{l}\text { mixed } \\
\text { on }\end{array}$ & H Towns ${ }^{\S}$ & R Towns` \\
\hline \multirow{2}{*}{34,8} & \multirow{2}{*}{3,96} & & & \multirow{2}{*}{34,37} & \multirow{2}{*}{6,55} \\
\hline & & $R=15,6$ & $H=17,66$ & & \\
\hline \multicolumn{2}{|c|}{$\begin{array}{l}\text { 8,78 times higher in } \\
\text { Hungarian population }\end{array}$} & \multicolumn{2}{|c|}{ Closest to the general rate } & \multicolumn{2}{|c|}{$\begin{array}{l}5.24 \text { times higher in towns } \\
\text { with Hungarian majority }\end{array}$} \\
\hline
\end{tabular}

\$-villages with exclusive Hungarian population;
\%-villages with exclusive Romanian population;
^-population ratio of aproximatively 1/1: R-romanians, H-hungarians;
\$-towns with Hungarian majority, aproximatively 60\%;
'-towns with almost exclusive Romanian population

Table 1 shows the big differences between half-closed rural communities with $\mathrm{R}$ or $\mathrm{H}$ population. There, $\mathrm{H}$ suicides are 8.78 times higher than $\mathrm{R}$. The difference between urban areas with a majority of $\mathrm{H}$ population and urban areas with almost exclusive $\mathrm{R}$ population is still very big: 5.24 times higher in $\mathrm{H}$. A fact worth noting is that rural and urban suicide rates in $\mathrm{H}$ communities are almost identical. This suggests the presence of a common contributing factor in $\mathrm{H}$ villages and towns regardless of education or cultural background.

Villages are half-closed communities that require a high degree of homogenous mentality, leading to a unique collective mentality of the entire community. From this point of view, rural settlements with a mixed $\mathrm{R}$ and $\mathrm{H}$ population of $1 / 1$ are closest to the general suicide rate of Bihor County. The influence proves to be mutual.

As seen above, the difference between suicide rates in $\mathrm{H}$ and $\mathrm{R}$ population within the same geographic area cannot be explained by the situational suicidal resonance. The suicide rate is similar in tendency on age groups, stages of biological vulnerability and gender difference. It differs, however, by accomplishment methods (involves imitation, trends and outlooks about suicide accomplishment). It differs as well by grouping the two populations in different collectivities with different religions and different We-ities. We call We-ity the spiritual, attitudinal and behavioral baggage of a populational group, as opposed to Alterity. On the other hand, the only contributing suicidal factors that could be pointed out were the cultural factor and the collective mentality of a community with its culture of suicide. These become part of what we call Culturally Vulnerable Suicide Areas.

\section{CONCLUSIONS}

○ The suicidal phenomenon has been slowly increasing for the past 10 years (12.04-17.44) in Bihor County, Romania, with natural situational fluctuations.

o The general 10 year suicide rate for Romanians was 11.30/100,000 and 21.65/100,000 for Hungarians. In almost exclusively Hungarian villages the suicide rate was about 9 times higher than in exclusively Romanian villages. In almost exclusively Hungarian cities the 
suicide rate was over 4 times higher than in almost exclusively Romanian cities. In mixed population towns close to 1:1 population ratio, the general suicide rate is included in the general fluctuation range.

- The only explanation found for the differences resulted from this study was the existence of a Culturally Vulnerable Suicide Area.

- These Cultural Vulnerable Suicide Areas require special monitoring so that principles of cultural prophylaxis of suicide could be issued.

- Influencing the collective mentality in this areas can only be done by an enlarged group consisting of psychiatrists, sociologists, psychologists, philosophers, teachers, priests and people of culture by engaging political factors, each of them using their own means of intervention in coordination with the other.

\section{PROPOSALS}

We suggest the WPA, in collaboration with national psychiatry associations, sociologists and psychologists, to initiate a project of registration of geographical areas with cultural vulnerability to suicide. Once registered, the common cultural issues of these areas could be understood, and thus we could proceed to the real prophylaxis of suicide.

\section{References}

Eibl-Eibesfeldt I (1995) Agresivitatea umană. (Krieg und Frieden aus der Sicht der Verhaltenforschung. Romanian re-edit) Bucharest: Ed. Treira, pp $29-35$.

Bertolote JM (2001) Foreword. In: De Leo D (ed.) Suicide and Euthanasia in Older Adults. Hogrefe and Huber Publisher. ed. v.i.

Jeffrey BA, Borbe RP, Birmaher B et al. (2005) Emergent suicidality in a clinical Psychoterapy Trial for Adolescent Depression. Am J Psychiatry 162: 2173 - 2175.

Buda M, Tsuang MT in Blumenthal SJ, Kupfer D (1990) Suicide over the life cycle. Washington: APA Press.

Durkheim E (1993) Despre sinucidere. Iaşi: Inst. European (1897. Le Suicide. Romanian re-edit).

Cozman D (2000) Sinuciderea. Cluj Napoca: Risoprint.

De Leo D (2001) Suicide and Euthanasia in Older Adults. Hogrefe and Huber Publisher, pp 21 - 37.

De Leo D, Bille-Brahe U, Kerkhof A et al. (2004) Suicidal Behaviour. Hogrefe and Huber, pp 17 - 93.

Kutcher S, Chehil S (2009) Suicide Risk Management. Lundbeck Institute, pp 80 - 87.

Potokar J, Thase M (2003) Advances in the Management and treatment of Depression. Martin Dunitz.

Schmidtke A, Bille-Brahe U, De Leo D, et al. (2004) Suicidal Behaviour In Europe. Hogrefe and Huber.

Scripcaru C (1996) Suicid şi agresivitate (Suicide and aggresivity). Bucharest: Psihomania.

Stroebe M, Stroebe W, Abakoumin G (2005) The Broken Heart: Suicidal ideation in Bereavment. Am J Psychiatry 162: 2178-2180.

Wasserman D (2001) Suicide - an unnecessary death. Martin Dunitz, pp 13-29. 